\title{
Acknowledgment to Reviewers of Universe in 2021
}

\section{Universe Editorial Office}

Citation: Universe Editorial Office. Acknowledgment to Reviewers of Universe in 2021. Universe 2022, 8, 86. https://doi.org/10.3390/ universe 8020086

Published: 29 January 2022

Publisher's Note: MDPI stays neutral with regard to jurisdictional claims in published maps and institutional affiliations.

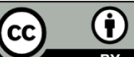

Copyright: (ㄷ 2022 by the author. Licensee MDPI, Basel, Switzerland. This article is an open access article distributed under the terms and conditions of the Creative Commons Attribution (CC BY) license (https://creativecommons.org/licenses/by/4.0/).

MDPI AG, St. Alban-Anlage 66, 4052 Basel, Switzerland

Rigorous peer-reviews are the basis of high-quality academic publishing. Thanks to the great efforts of our reviewers, Universe was able to maintain its standards for the high quality of its published papers. Thanks to the contribution of our reviewers, in 2021, the median time to first decision was 17 days and the median time to publication was 35 days. The editors would like to extend their gratitude and recognition to the following reviewers for their precious time and dedication, regardless of whether the papers they reviewed were finally published:

Abbrescia, Marcello

Abouelmagd, E. I.

Agarwal, Nishant

Agathos, Alexander

Agop, Maricel

Aguerri, José Alfonso

Akcay, Sarp

Akhmedov, Emil

Akiyama, Kazunori

Alessi, Elisa Maria

Alexeyev, Stanislav

Alexiou, Spiros

Allen, Branden T.

Alonso-Serrano, Ana

Altschul, Brett

Altwegg, Kathrin

Alvarez-Castillo, David Edwin

Alves, Marcio

An, Haipeng

Anastopoulos, Charis

Andersen, Thomas

Anguelova, Lilia

Antonelli, Marco

Appleby, Stephen

Armoni, Adi

Arnould, Marcel

Aros, Rodrigo

Arraut, Ivan

Arshakian, Tigran G.

Artymowski, Michal

Arvanitakis, Alex S.

Arzano, Michele

Asorey, Jacobo

Aste, Andreas
Knorr, Benjamin

Kocharyan, Armen A.

Kohri, Kazunori

Kole, Merlin Reynaard

Kološ, Martin

Komissarov, Serguei S.

Konishi, Kenichi

Kosinski, Piotr

Kosmas, Theocharis

Kotikov, Anatoly

Kotila, Jenni

Kraniotis, Georgios V.

Kratz, Karl-Ludwig

Krssak, Martin

Kuin, Paul

Kun, Emma

Kunz, Martin

Laha, Sibasish

Laliotis, Athanasios

Lapi, Andrea

Latosh, Boris

Leahy, Denis

Lebiedowicz, Piotr

Lee, Chien-Hsiu

Lee, Hyun Su

Leisos, Antonios

Lenske, Horst

León, Alejandro

Leontaris, George K.

Lepe, Samuel

Lesur, Maxime

Lev, Felix M.

Li, Bo

Li, Jian 
Atchison, Justin A.

Attie, David

Avelino, Pedro

Aviles, Alejandro

Awasthi, Arun Kumar

Ayriyan, Alexander

Badziak, Marcin

Balázs, Heilig

Baldo, Marcello

Bamba, Kazuharu

Barabash, Alexander

Barak, Liron

Barbaro, Maria B.

Barbero, Fernanado

Barboza, Edésio Miguel

Bargueno, Pedro

Barnich, Glenn

Barrau, Aurelien

Bartelmann, Matthias

Batista, Rafael Alves

Baù, Giulio

Baum, Sebastian

Bazilevskaya, Galina Alexandrovna

Beesham, Aroonkumar

Behunin, Ryan

Bellini, Gianpaolo

Beltran Jimenez, Jose

Benedetti, Dario

Benetti, Micol

Benkő, József

Beraudo, Andrea

Berezin, Victor Alexandrovich

Berger, Niklaus

Bergeron, Hervé

Berton, Marco

Bettoni, Daniela

Bhattacharyya, Trambak

Biesiada, Marek

Bini, Donato

Bisnovatyi-Kogan, Gennady S.

Bobomurat, Ahmedov

Bonanno, Alfio

Bonatsos, Dennis

Bonometto, Silvio A.

Bonora, L.

Bonventre, Richard

Bopp, Fritz W.

Bordag, Michael

Borges, Marcelo

Borisova, T. D.

Borka, D.
Li, Xiaocan

Li, Yufeng

Liakos, Alexios

Liang, Shidong

Licata, Ignazio

Liegener, Klaus

Lin, Chia-Min

Lin, Guey-Lin

Lin, Lap-Ming

Linares, Roberto

Ling, Yi

Linhares, César

Link, Jonathan

Liu, Fuhu

Liu, N.

Liu, Xiang

Liukkonen, Jukka

Lobato, Ronaldo

Lobo, Iarley

Lobo, Iarley Pereira

Loewe, Marcelo

Logoteta, Domenico

Lombriser, Lucas

López-Moya, Marcos

Lovell, Mark

Luciano, Giuseppe Gaetano

Lucini, Biagio

Lukierski, Jerzy

Luning, Sebastian

Luongo, Orlando

Luque-Escamilla, Pedro L.

Lyakhov, Andrey

Lyutikov, Maxim

Macedo, Caio

Maeder, Andre

Magdy, Niseem

Malek, Emanuel

Malgieri, Massimiliano

Malkiewicz, Przemyslaw

Malkov, Oleg

Maltseva, O. A.

Malyshev, Denys

Mamun, A. A.

Marachevsky, Valery

Marciano, Antonino

Margalit, Ben

Mariani, Camillo

Marle, Charles-Michel

Marochnik, Leonid

Martello, Daniele

Martí, Josep 
Bormashenko, Edward

Bornyakov, Vitaly

Borowiec, Andrzej

Bouwmeester, Dirk

Brahma, Suddhasattwa

Branca, Antonio

Brandenberger, Robert $\mathrm{H}$.

Brandt, Bastian

Bressan, Alessandro

Brevik, Iver $\mathrm{H}$.

Brose, Robert

Brugnera, Riccardo

Buchert, Stephan C.

Buchholz, Detlev

Burdin, Sergey

Burgdorf, Martin

Busso, Maurizio M.

Caceres, Elena

Cahay, Marc M.

Cai, Qingyu

Calloni, Enrico

Cander, Ljiljana R.

Cao, Junjie

Cao, Zhen

Capistrano, Abraao J. S.

Capozziello, Salvatore

Cappelluti, Nico

Cappuzzello, Francesco

Cardone, Fabio

Carpano, Stefania

Casadio, Carolina

Casadio, Roberto

Caso, Loredana

Casolino, Marco

Cassani, Davide

Cattaneo, Paolo W.

Cavalcanti, Rogerio Teixeira

Cei, Fabrizio

Cella, Giancarlo

Cembranos, Jose A. R.

Ceriotti, Matteo

Cerri, Silvio Sergio

Cervantes-Cota, Jorge

Cesarini, Andrea

Chakraborty, Nachiketa

Char, Prasanta

Chatwin-Davies, Aidan

Chatzistavrakidis, Athanasios

Chen, Che-Yu

Chernodub, Maxim

Chernyshov, Dmitrii O.
Martín, Eduardo L.

Martín-Albo, Justo

Martin-Benito, Mercedes

Martineau, Killian

Martino, Ivan De

Martucci, Matteo

Marziani, Paola

Marzola, Luca

Maslov, Konstantin A.

Mastrolia, Pierpaolo Mastrolia

Masullo, Maria Rosaria

Mateos Guilarte, Juan

Matsuno, Ken

Matteo, Armando Di

Mattingly, David

Mavromatos, Nick E.

Mavromichalaki, Helen

Mayerson, Daniel R.

Mazin, Daniel

Mazzitelli, Francisco Diego

McCollough, Michael

Melkikh, Alexey V.

Melnikov, Alexey A.

Mena-Barboza, Eri A.

Mena-Marugan, Guillermo A.

Menci, Nicola

Menéndez, Javier

Mennickent, Ronald

Meyer, Dominique

Meyer, Manuel

Micol, Benetti

Mielke, Eckehard

Mignani, Roberto

Miković, Aleksandar R.

Millington, Peter

Milton, Kimball A.

Mimoso, José $P$.

Minkevich, Albert

Miralles, Juan Antonio

Miranda, Omar G.

Miscetti, Stefano

Mitchell, Alison

Mohr, Peter

Moniz, Paolo Vargas

Moore, Christopher J.

Morikawa, Masahiro

Morlino, Giovanni

Morozumi, Takuya

Moshin, Pavel Yu.

Mosquera Cuesta, Herman J.

Moulin, Emmanuel 
Chervon, Sergei

Chiofalo, Maria Luisa

Chiosi, Cesare

Chodos, Alan

Choudhury, Sayantan

Cirigliano, Vincenzo

Claude, Semay

Claudi, Riccardo

Cleaver, Gerald

Co, Raymond T.

Cohen, Thomas

Colas, Paul

Colgain, Eoin

Colgáin, Eoin Ó.

Colò, Gianluca

Colón, Cristóbal

Combrinck, Ludwig

Correa, Diego

Cortes, Jose

Costa, João Lopes

Costa, L. Filipe O.

Couchot, Francois

Coughlin, Michael

Cremaschini, Claudio

Criss, Robert E.

Cristallo, Sergio

Cristea, Irina

Cristofari, Pierre

Cruz Osorio, Alejandro

Cui, Wei

Curiel, Erik

Cusin, Giulia

Cvetkovic, Branislav

Czerny, Bozena

Czuchry, Ewa

Dabrowski, Mariusz

Damiani, Francesco

Darmora, Smita

Dasgupta, Keshav

Datta, R.

Dave, Romeel

Davidson, Aharon

De Angelis, Alessandro D.

De Araujo, Jose Carlos Neves

De Diego Onsurbe, José Antonio

De Freitas-Pacheco, José Antonio

De Holanda, Pedro Cunha

De Jesus, José Fernando

De La Peña, Luis

De Martino, Ivan

De Mello, Eugênio Ramos Bezerra
Mourenas, D.

Moustakidis, Charalampos

Mueck, Wolfgang

Munch, Jesper

Mushtukov, Alexander

Nagasawa, Michiyasu

Nagirner, Dmitrij I.

Nakamura, Atsushi

Nara, Yasushi

Natário, José

Nathanail, Antonios

Navarro-Salas, Jose

Nedkova, Petya G.

Neronov, Andrii

Neuhäuser, Ralph

Neves, J. C. S.

$\mathrm{Ng}$, Alfred

Nguyen, Ann

Nicastro, Luciano

Nicolas, Chamel

Nigro, Cosimo

Nikolaevich, Frolov Boris

Nikulov, Alexey V.

Nishikawa, Ken-Ichi

Nogueira, Flavio S.

Nojiri, Shin'ichi

Nordhaus, Jason

Nowakowski, Marek

Nozzoli, Francesco

Nunes, Nelson

Nunes, Rafael C.

Nurmagambetov, A. J.

Nuth, Joseph A.

Obukhov, Yuri

Odintsov, Sergei

Oliveira, Fernando

Oliveri, Roberto

Orazi, Emanuele

Orlandini, Mauro

Ortega, Pablo G.

Osetrin, Konstantin

Oshita, Naritaka

Ovalle, Jorge

Ovgun, Ali

Oxman, Luis E.

Ozaydin, Fatih

Pagano, Davide

Pagliara, Giuseppe

Pagnanini, Lorenzo

Paic, Guy

Pais, Helena Sofia 
De Paolis, Francesco

De Sanctis, Maria Cristina

Decca, Ricardo S.

Dedkov, G. V.

Degl'Innocenti, Scilla

Degollado Daza, Juan Carlos

Degollado, Juan Carlos

Del Popolo, Antonino

Del Sordo, Stefano

Dell'Agli, Flavia

Dembinski, Hans

Deming, L. Drake

Demura, Alexander

Deppman, Airton

Deriglazov, Alexei

Descouvemont, Pierre

Di Crescenzo, Antonia

Di Fillipo, Francesco

Di Giacomo, Adriano

Di Mauro, Marco

Di Valentino, Eleonora

Dialektopoulos, Konstantinos

Diamantini, Maria Cristina

Dieks, Dennis

Dimitrijević, Milan S.

Dimopoulos, Konstantinos

Dmitry, Blinov

Dokuchaev, Vyacheslav

Dolgov, Alexander

Domi, Alba

Doneva, Daniela D.

Doroshkevich, Andrey

Dubeibe, Fredy L.

Duranti, Matteo

Dybalski, Wojciech

Dymnikova, Irina

Ellerman, David

Elsaesser, Dominik

Entem, David R.

Esquivel-Sirvent, R.

Etesi, Gabor

Ettori, Stefano

Exirifard, Qasem

Fabbri, Luca

Fabregat, Juan

Falciano, F. T.

Falls, Kevin G.

Farres, Ariadna

Favole, Ginevra

Feng, James $Q$.

Feoli, Antonio
Pais, Pablo

Pala, Francesca

Palasantzas, George

Palma, Francesco

Palmerini, Sara

Pamucar, Dragan

Panotopoulos, Grigoris

Papaderos, Polychronis

Papadimitriou, Constantinos

Papaioannou, Athanasios

Papitto, Alessandro

Pappas, George

Parkinson, David

Parotto, Paolo

Partouche, Hervé

Paschos, Emmanuel A.

Passek-Kumeriçki, Kornelija

Passon, Oliver

Pastore, Alessandro

Paul, Tanmoy

Paulos, Miguel F.

Paunzen, Ernst

Pavón, Diego

Pegoraro, Francesco

Percacci, Roberto

Pereira, J. G.

Pereira, S. H.

Peres, Orlando

Perez, Alejandro

Perivolaropoulos, Leandros

Perrino, Roberto

Peter, Patrick

Petroni, Nicola Cufaro

Petrov, Albert

Petruzziello, Luciano

Pezzella, Franco

Piatti, Andres E.

Picanço, Rodrigo

Piemontese, Stefano

Pierrehumbert, Raymond

Pignalberi, Alessio

Pikuz, Tatiana

Pillepich, Annalisa

Pinčák, Richard

Pirozhenko, Irinia

Piscoran, Laurian-Ioan

Pithis, Andreas G. A.

Pitkin, Matthew D.

Platania, Alessia

Podgornik, Rudi

Poedts, Stefaan 
Fermi, Davide

Fernández, Francisco

Fernández-Ontiveros, Juan A.

Ferraro, Rafael

Ferreira, Márcio

Ferri, Elena

Fidaleo, Francesco

Fields, Chris

Figueiredo, Cosme Alexandre O. B.

Filippov, Anatoly Vasilievich

Fillion-Gourdeau, François

Finoguenov, Alexis

Flachi, Antonino

Fomin, Igor Vladimirovich

Fortes, Elaine C. F. S.

Foschini, Luigi

Foster, Adam

Friedman, Yaakov

Fujiwara, Mamoru

Gadella, Manuel

Gail, Hans-Peter

Gaite, Jose

Gambacurta, Danilo

Gao, Changjun

Gao, Sijie

Garain, Sudip K.

Garay, Luis J.

García Aspeitia, Miguel Angel

Garcia De Andrade, Luiz

García-Compean, Hugo

Garcia-Hernandez, Domingo Anibal

Gasparrini, Dario

Gasperini, Maurizio

Gavrilov, Boris

Gavrilov, Sergey

Geng, Chaoqiang

Gerdjikov, Vladimir Stefanov

Gerontidou, Maria

Giammarchi, Marco

Gianotti, Paola

Gieres, François

Gil-Świderska, Agnieszka

Giordano, Matteo

Glushkov, Alexander V.

Gnecchi, Alessandra

Godłowski, Włodzimierz

Golovnev, Alexey

Gómez, Diego Sáez-Chillón

Gómez-Valent, Adrià

González García, Maria Concepción

González Romero, Luis Manuel
Poggiani, Rosa

Polarski, David

Polonyi, Janos

Polosa, Antonio D.

Ponomarev, Dmitry

Popa, Lucia Aurelia

Popov, Arkadiy

Popov, Sergei B.

Portillo, Israel

Pozdeeva, Ekaterina O.

Preka-Papadema, Panagiota

Providencia, Constança

Pshirkov, Maxim

Pushkarev, Alexander

Qi, Chong

Quartin, Miguel

Radinschi, Irina

Raffelt, Georg

Raggi, Mauro

Rajendra, Gupta

Ramos, Angels

Rashkov, Radoslav

Rastgoo, Saeed

Ratti, Claudia

Rebouças, Marcelo

Reichert, Manuel

Ricci, Fulvio

Richarte, Martín G.

Rincón, Ángel

Ring, Peter

Rizos, John

Robertson, Hamish

Rocha, Jorge

Rodrigues, Davi

Rodriguez, Manuel

Rodriguez-Lopez, Pablo

Rogatko, Marek

Romano, Vittorio

Romanyuk, Iosif

Ronga, Francesco

Roshchupkin, Sergei P.

Rosmej, Frank

Rott, Carsten

Rovelli, Carlo

Rubiera-Garcia, Diego

Rudenko, Sergei

Rueda, Jorge

Ruiz-Granados, Beatriz

Rybczyński, Maciej

Sá, Paulo M.

Sabido, Miguel 
Good, Michael R. R.

Goodsell, Mark

Gorbunov, Dmitry

Gordon, Christopher

Goudarzi, Mohammad Ali

Gourgouliatos, Konstantinos

Grassi, Pietro Antonio

Graziani, Luca

Greensite, Jeffrey Paul

Grib, A. A.

Grøn, Øyvind

Grunfeld, Ana Gabriela

Guendelman, Eduardo

Guimarães, Marcelo

Gulminelli, Francesca

Guo, Fengkun

Guo, Minyong

Guo, Yiqing

Gupt, Brajesh

Gusakov, Mikhail

Guzmán, María José

Hamber, Herbert W.

Hands, Simon

Harabasz, Szymon Piotr

Harremoës, Peter

Hawton, Margaret

Hayakawa, Takehito

Hayashi, Keiji

Heinemann, Stephan G.

Helayel-Neto, J. A.

Helayel-Neto, Jose

Held, Aaron

Henriksen, Mark

Hervet, Olivier

Hirotani, Kouichi

Hochlaf, Majdi

Hoelbling, Christian

Hoffmann, Andreas

Hofmann, Ralf

Hofmeister, Anne

Hohmann, Manuel

Homola, Piotr

Hong, Byungsik

Horatiu, Nastase

Horn, Bart

Høye, Johan

$\mathrm{Hu}$, Bin

Huang, Feifei

Hugbart, Mathilde

Ichiki, Kiyotomo

Iguchi, Hideo
Sabín, Carlos

Sahlén, Martin

Sahoo, Pradyumn Kumar

Said, Jackson Levi

Salafia, Om

Salucci, Paolo

Sanchez Contreras, Carmen

Sanchez, Norma G.

Sánchez, Sebastian F.

Santopinto, Elena

Santos, Alesandro

Sapone, Domenico

Saridakis, Emmanuel

Saridakis, Manos

Sasakura, Naoki

Satz, Helmut

Savelova, Elena

Scarr, Tzvi

Schäfer, Gerhard

Schettino, Giulia

Schmidt, Michael A.

Schmieder, Brigitte

Schoeffler, Kevin

Schoppmann, Stefan

Schreck, Marco

Schröder, Stefan

Sebastiani, Lorenzo

Secrest, Jeffery

Sedaghati, Elyar

Sedrakian, Armen

Seo, Min-Seok

Sesana, Alberto

Sgalaberna, Davide

Sgouros, Onoufrios

Shankar, Karthik H.

Shapiro, Ilya

Shapiro, Irwin

Sharov, German Sergeevich

Shen, Fang

Sheykin, Anton A.

Shi, Jianrong

Shore, Graham

Shtanov, Yuri

Silva, Marcos V. De S.

Simpson, Alex

Singleton, Douglas

Sinitsyna, Vera

Smirnov, Alexei

Smolić, Ivica

Sneden, Chris

Sofue, Yoshiaki 
Impens, Francois

Inagaki, Tomohiro

Incicchitti, Antonella

Ingold, Gert-Ludwig

Iorio, Alfredo

Iorio, Lorenzo

Itaco, Nunzio

Ivanov, Martin V.

Jana, Sudip

Janssen, Oliver

Järv, Laur

Jawad, Abdul

Jiang, Biwei

Jokela, Niko

Jonke, Larisa

Jora, Renata

Jordi, Carme

Joung, Euihun

Junker, Georg

Jusufi, Kimet

Kalantonis, Vassilis

Kallunki, Juha

Kamenshchik, A. Yu

Kaminker, Alexander D.

Kannawadi, Arun

Karbstein, Felix

Karch, Andreas

Kasetti, Siva Prasad

Kashi, Amit

Katsova, Maria

Kazanas, Demosthenes

Keppens, Arno

Kerner, Richard

Ketov, Sergei

Khan, Amir N.

Khanna, Faqir C.

Khlopov, Maxim

Khurshudyan, Martiros

Khusnutdinov, Nail

Kieda, David

Kim, Choong

Kim, Dong-Woo

Kim, Nakwoo

Kirillov, Alexander

Kirschner, Roland

Kislov, Roman

Kisslinger, Leonard S.

Kitamoto, Shunjii

Kleidis, Kostas

Klimenko, Maksim V.

Knecht, Andreas
Sohn, Jubee

Sokoloff, Dmitry

Solà, Joan

Soler, Paul

Soma, Arun Kumar

Sonnino, Giorgio

Sorriso-Valvo, Luca

Sparvoli, Roberta

Spurio, Maurizio

Spyrou, Nikolaos K.

Stachurska, Juliana

Staicova, Denitsa

Stamerra, Antonio

Starobinsky, Alexei A.

Stasielak, Jarosław

Staykov, Kalin V.

Steffen, Gielen

Steinheimer, Jan

Stenflo, Lennart

Stoica, Sabin

Stornaiolo, Cosimo

Stratta, Giulia

Strong, Andrew W.

Sultana, Joseph

Sushkov, Sergei

Tan, Baolin

Tang, Qingwen

Tartaglia, Angelo

Tedesco, Luigi

Teixeira Alves, Danilo

Terranova, Francesco

Tessarotto, Massimo

Thompson, David J.

Titov, Arsenii

Tiwari, Prabhakar

Tiwari, Shubhanshu

Tomasiello, Alessandro

Tomassetti, Nicola

Tommei, Giacomo

Toribio, Alan Miguel Velasquez

Triantafyllopoulos, Alkiviadis

Trushkin, Sergei

Turimov, Bobur

Typel, Stefan

Urban, Michael

Valcarcel, Jorge Gigante

Vallenari, Antonella

Van De Meent, Maarten

Van de Steene, Griet C.

Van Den Heuvel, Edward P. J.

Vandevender, John Pace 
Varekamp, Johan

Varieschi, Gabriele U.

Vasko, Ivan Y.

Vassilevich, Dmitri

Vazquez, J. Alberto

Velten, Hermano

Ventura, Paolo

Veres, Péter

Vernieri, Daniele

Villaseñor, Eduardo J. S.

Vilmer, Nicole

Vink, Jacco

Visinelli, Luca

Visser, Manus

Vitiello, Giuseppe

Von Marttens, Rodrigo

Wagner, Fabian

Wagner, Jenny

Waldron, Abbey

Wang, Anzhong

Wang, Gaige

Wang, Qiliang

Wang, Shao-Jiang

Wascko, Morgan

Weber, Fridolin

Weissman, Dorin

Wette, Karl

Wiita, Paul

Williams, David A.
Winstanley, Elizabeth

Wong, Chik Him

Wysokinski, Karol I.

Xie, Fuguo

$X u$, Renxin

Xu, Rui

Yamada, Masatoshi

Yates, Jeremy A.

Yeom, Dong-han

Yoshii, Ryosuke

Youssef Moussa, Miled Hassan

Yungelson, Lev R.

Zabrodin, Evgeny

Zakharov, Alexander

Zhang, Baocheng

Zhang, Fan

Zhang, Guohui

Zhang, Haocheng

Zhao, Hongsheng

Zhong, Jiahao

Ziaeepour, Houri

Zijlstra, Albert

Zimovets, Ivan

Zivieri, Roberto

Zong, Qiugang

Zubkov, Mikhail

Zugec, Peter

Zyuzin, Dmitry A. 